\title{
La cultura como eje transversal en el aprendizaje basado en tareas: reflexiones y sugerencias para la enseñanza del español como segunda lengua ${ }^{1}$
}

\author{
Luis Guillermo Barrantes² \\ Universidad Nacional, Costa Rica
}

\section{RESUMEN}

Se presenta un modelo de enseñanza del español como segunda lengua o como lengua extranjera mediante la metodología del aprendizaje basado en tareas (ABT). Se enlaza tal metodología con la inclusión del tema de la cultura como eje transversal. Se describe como ejemplo una tarea de una unidad de determinado curso, a fin de que el docente de Español perciba los alcances del plan propuesto.

\section{Abstract}

A model of Spanish as a second/foreign language teaching using a task-based methodology is presented here. Likewise, culture is included as a cross-curricular topic. An example is given of a task which is part of a unit of a specific course, with the purpose of enabling an instructor of Spanish to visualize the scope of the plan proposed here.

Palabras clave: enseñanza de lenguas extranjeras, enseñanza del español, metodología de la enseñanza, aprendizaje basado en tareas. Keywords: foreign language teaching, Spanish as a second language, teaching methodology, task-based approach.

\footnotetext{
${ }^{1}$ Recibido: 9 de abril 2008; aceptado: 30 de mayo 2009.

${ }^{2}$ Correo electrónico: luisba66@yahoo.es
} 


\section{Introducción}

Pese a que durante mucho tiempo en varios países se ha enseñado español como segunda lengua (ESL) o como lengua extranjera (LE) y a la bibliografía publicada sobre la materia, en Costa Rica se podría afirmar que ese tema es aún incipiente. Los primeros intentos documentados de enseñanza de ESL/LE en Costa Rica se remontan a principios de la década de 1970, en escuelas privadas, algunas de las cuales aún existen. Es encomiable la labor que tales instituciones han realizado no sólo en la capacitación de sus profesores sino también en la producción de materiales. Tales instituciones han desarrollado además enfoques particulares y característicos según sus intereses dentro del marco comunicativo (por ejemplo, la conversación y el entrenamiento de personal misionero). Si bien las academias de idiomas han desempeñado un papel pionero, también su personal docente se ha profesionalizado muy poco a poco a partir de una práctica empírica e incluso artesanal. Justo es destacar que ese personal ha buscado la profesionalización por sus propios medios y por su propio interés.

Pasados muchos años de ofrecer en el extranjero toda clase de cursos de español, pese a la limitación en cuanto a materiales didácticos y preparación de los docentes, se inicia en las universidades costarricense - en la década de 1990 - un proyecto para sistematizar la preparación de los docentes mediante la apertura de programas de Maestría en la Enseñanza de ESL/LE y de Educación con énfasis en la enseñanza de ESL/LE. Los participantes que reclutan estos programas son en su mayoría profesores de los institutos privados con experiencia adquirida en la práctica en cada instituto, que desean sistematizarla y adquirir nuevas metodologías en las tendencias de enseñanza de idiomas. También hay quienes provienen de la enseñanza del español como lengua materna.

Gracias a que paulatinamente se han importado distintos texto y manuales, se posee hoy un útil acervo bibliográfico. Sin embargo, la principal fuente de apoyo es el internet y la voluntad de compartir 
teoría, práctica e investigación que la comunidad académica internacional se esfuerza por mantener. Por ejemplo, se cuenta con el Marco Común Europeo de Referencia para la Enseñanza de las Lenguas (MCEREL) y los recursos que entidades como el Instituto Cervantes de España ${ }^{3}$ ponen a disposición de los capacitadores de docentes y de los docentes en formación.

El presente artículo procura contribuir con esa comunidad académica desde la experiencia de la enseñanza de ESL/LE en la Universidad Nacional de Costa Rica según el enfoque de Aprendizaje Basado en Tareas (ABT). Para su aplicación se han hecho adaptaciones del Task-Based Approach (TBA) empleado en la enseñanza del inglés como segunda lengua ${ }^{4}$.

\section{¿Qué es el aprendizaje basado en tareas?}

Conviene hacer referencia a lo que especialistas en adquisición de una segunda lengua denominan «el complejo de Atlas»; una tendencia a desarrollar las clases con una metodología centrada en sí mismos en cuanto «poseedores» del conocimiento y cuya función consiste en transmitirlo a los alumnos. Por ello, el papel desempeñado por el docente y el alumno definía las tareas por realizar. Se asumía que cuanto mejor fuera la calidad de las explicaciones del docente, más aprenderían los alumnos; cuanto mejores fueran las preguntas formuladas por aquél, mejores serían las respuestas de éstos. Estas premisas están presentes en cualquier metodología de clase que se asemeje al método audiolingualista. Se hace uso de la analogía de

\footnotetext{
3 Véase el Marco Común Europeo para la Enseñanza de las Lenguas (MCEEL) en $<\mathrm{http} / / \mathrm{www}$.institutocervantes.com>.

${ }^{4}$ Jack Richards y Theodore S. Rodgers, Approaches and Methods in Language Teaching (Cambridge: Cambridge University Press, 2001) cap. 18.

${ }^{5}$ Atlas, en la mitología griega, corresponde a un titán condenado a sostener los cielos sobre sus espaldas. Con este término se designa también a cualquier persona que soporta un gran peso. Para profundizar en el asunto, ver James F. Lee y Bill Van Patten, Making Commnicative Language Teaching Happen (Nueva York: McGraw Hill, 1995) cap. 1.
} 
Atlas porque el docente echa sobre sus espaldas toda la responsabilidad de lo que ocurre en la clase. Tal metodología es muy antigua; desde la escolástica medieval solía decirse irónicamente «omne autem quod recipitur, ad modum recipientis recipitur» (Todo lo que se recibe se recibe al modo del recipiente). Con ello se indicaba que el alumno aprende sólo lo que el docente deposita en su mente, una falla en el aprendizaje seguro se debía a la carencia del alumno para recibir el conocimiento, nunca del docente, poseedor de la verdad.

Con el nacimiento del método comunicativo para la enseñanza de idiomas, la situación varió. Los papeles de profesor y el alumno también y con más participación espontánea por parte del alumno. Aun así, el cambio no fue suficiente. Tanto profesores como alumnos aceptaban el hecho de que los docentes eran los expertos y, por tanto, debían ser las figuras centrales en la clase. Por ello el profesor conservaba aún su papel entrenador de los alumnos en los hábitos apropiados de aprendizaje y de guardián de la precisión en el manejo del idioma.

La liberación de Atlas del peso que había soportado, en el caso de los docentes, se dio con un cambio drástico en la percepción del proceso enseñanza-aprendizaje. Es decir, el cambio se suscitó en el momento en que las tareas por realizar empezaron a delimitar los papeles tanto del docente como de los alumnos. A partir de entonces se comenzó a ver al docente como un recurso para los alumnos y como un arquitecto.

Al adoptar su nuevo papel de recurso para los alumnos, el docente se comporta en clase como lo haría si estuviera en la calle ayudando a alguien a entender una dirección; quien da una dirección a un desconocido asume que su interlocutor comprende lo que se le dice y sólo hará especificaciones o aclarará cosas en la medida en que requiera y solicite. No se siente responsable desde el inicio de garantizar si el que consultó está asimilando la información. En el ambiente de clase, el alumno tiene un papel de sujeto activo. Sin embargo, tampoco es el centro de la clase. Lo que está en el centro es 
el proceso mismo del aprendizaje y la tarea concreta por realizar. Cuando se logra, los alumnos se independizan y la interacción entre ellos es mucho más enriquecedora; aprenden a negociar el significado de las cosas y a percibir al docente como un recurso, entre otros.

Para comprender al docente en su papel de arquitecto, veamos también lo que pasa fuera de clase. En una conversación entre varios interlocutores, no existe un individuo que asigne los turnos de intervención; todos participan espontáneamente. Alguien puso el tema y luego todos lo desarrollan hasta un desenlace. El docente que asume el papel de arquitecto es aquel que diseña y planea una tarea, pero no se siente responsable del producto final. Los estudiantes son colaboradores y constructores de la tarea.

Cuando el docente planea una tarea, tiene en cuenta el nivel de desempeño de los alumnos, de modo que la realización de esa tarea pueda sumar algo a la adquisición del idioma que el estudiante ya posee, pero que no exceda demasiado lo que el alumno está en capacidad de hacer. También debe estar en capacidad para establecer espacios adecuados para proveer instrucción sobre errores detectados; ésta puede ser una actividad en la que todos tengan participación activa. Si se lleva cuenta de los errores cometidos durante las intervenciones de los estudiantes, se pueden diseñar actividades en las que los mismos alumnos sugieran las correcciones en la medida de lo posible.

El aprendizaje basado en tareas es, pues, un modo de aprender en el cual el docente, despojado de su papel autoritario y poseedor del conocimiento, prepara tareas en las que los alumnos se involucren plenamente y construyan el conocimiento por sí mismos.

\section{¿Cómo combinar un eje transversal en un curso de ESL/LE con la metodología ABT?}

El propósito de los programas de estudio en el extranjero para estudiantes universitarios no es solamente la adquisición o el perfeccionamiento del dominio de un idioma. Se busca que los 
jóvenes, cuya oportunidad de salir de su país e internarse en nuevas culturas es una oportunidad que la mayoría del mundo no tiene, asuman la experiencia como una responsabilidad para su propia visión del mundo y para la construcción de más altos niveles de conciencia en su futuro desempeño profesional. Por esa razón, parece conveniente que los cursos de español que se diseñan para esos jóvenes estén permeados por un componente de cultura de la comunidad anfitriona, además del componente de lenguaje que vienen a estudiar.

Ese componente de cultura, o eje transversal, enlaza los distintos factores del currículo, de manera que en el curso no se desarrollen actividades comunicativas en función del lenguaje aprendido sino el empleo del lenguaje como facilitador de la experiencia cultural. Para ello, se requiere que los contenidos del curso deben plantearse y distribuirse a modo de tareas que el estudiante debe ejecutar para incrementar su desempeño en el idioma, a la vez que conoce la cultura.

\section{¿Qué es un eje transversal?}

Dentro del planeamiento curricular se entiende por eje transversal un tema definido que articula el contenido del curso en su totalidad. En el caso de ABT para un curso de ESL/LE, cada tarea y sus distintas etapas (previo, durante, y posterior a la tarea), se diseñan conforme a ese eje. La novedad es que el eje transversal da la pauta para la selección de contenidos, y no a la inversa. Si bien un curso de cualquier disciplina puede plantearse en torno a un eje transversal, más sentido tiene hacerlo en un curso en que los estudiantes aprenden un idioma en un país y en una cultura que no son los suyos. Aprender el idioma per se no es de mucho valor si tal aprendizaje no está contextualizado dentro de la cultura donde se habla. Las «cápsulas culturales» que suelen incluirse en los cursos, no subsanan la deficiencia; más bien la exponen. 
Para que los objetivos del curso ABT se logren de modo satisfactorio, se deben establecer parámetros adecuados de evaluación formativa y sumativa que permitan tener una idea objetiva del aprovechamiento de la experiencia y el desempeño en el lenguaje que cada alumno ha alcanzado ${ }^{6}$.

\section{¿Cómo seleccionar los contenidos en torno a la cultura como eje transversal?}

La manera tradicional de establecer los contenidos de un curso ESL/LE consiste en ordenar una lista de temas gramaticales de modo que cada uno suponga el precedente. Aquí proponemos un modelo que genere los contenidos del curso a partir de la misma experiencia de aprendizaje. Esto hace que los estudiantes estén plenamente involucrados en la selección de contenidos.

El primer paso del docente es conocer el nivel de desempeño en español que muestran los estudiantes antes de iniciar del curso; esto se logra mediante pruebas de diagnóstico. Cuanto mayor sea el dominio del idioma mostrado por los estudiantes, mejor será su participación en la discusión de los contenidos. Una vez determinado ese nivel y tomando las previsiones necesarias en cuanto a lo que pueden hacer, se les invita a una «lluvia de ideas» sobre temas culturales acerca de los cuales les gustaría hacer investigaciones. Estas investigaciones no serán meramente bibliográficas sino producto de entrevistas en la comunidad. Los estudiantes escogerán un tema, elaborarán un cuestionario y se entrevistan con distintas personas para elaborar un informe oral y escrito acerca de los resultados obtenidos. En algunos grupos de estudiantes se da una gran facilidad para

${ }^{6}$ Alice C. Omaggio entiende los conceptos de formación formativa y sumativa así: «formative evaluation - a type of evaluation that enables teachers to alter instruction in progress to better address the needs of the learners»; summative evaluation-proficiency tests that measure «the language abilities of individuals at a given point in time, without regard to a specific course of instruction», Teaching Language in Context (Boston: Heinle \& Heinle, 1986) 312. 
proponer temas; en otros casos, el docente debe sugerir algunos a modo de ejemplo para que los alumnos piensen en otras posibilidades.

El primer informe de investigación debe presentarse a la mayor brevedad posible; por ejemplo, una semana después de haberse asignado los temas. Tanto los informes orales como los escritos darán la materia prima inicial para la selección de contenidos de lenguaje. En el caso de los informes escritos, el docente puede elegir algunos fragmentos de los informes que contengan errores o imprecisiones muy recurrentes o significativas para la comunicación. En cuanto a las presentaciones orales, el docente deberá grabar las participaciones o tomar nota de los errores más visibles. Durante la siguiente clase de

las presentaciones, el profesor distribuirá ejemplos con frases $\mathrm{u}$ oraciones con errores recopilados. Tales errores pueden estar señalados o resaltados por el profesor, pero no corregidos, a fin de que sean los estudiantes quienes propongan la corrección.

A partir de entonces, los estudiantes comenzarán a enumerar una serie de temas de cohesión léxica o gramatical que se deberán estudiar en las clases sucesivas. Con esta materia prima, se podrán preparar actividades en las que los estudiantes propongan la corrección de las imprecisiones detectadas. De esa manera, los estudiantes acumulan generalizaciones y reglas que deberán sistematizar con ayuda del profesor para que a final del curso cuenten con un compendio de funciones léxicas y gramaticales, como referente en el futuro ${ }^{7}$. Además, el profesor puede usar ese material como fuente para el diseño de otras tareas en las que los estudiantes realicen algún

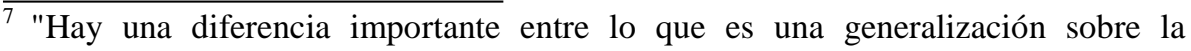
gramática y lo que es una regla gramatical. Por ejemplo, hay una regla en inglés según la cual «there is» aparece delante de sustantivos en singular $\mathrm{y}$ «there are» delante de sustantivos en plural: There is one vs. There are two. Sin embargo, se oyen con frecuencia frases como «There's ants in the kitchen», o «Take one; there's a lot». La generalización lingüística dice que se elige la forma singular del verbo porque no hay un sustantivo anterior que rija el número del verbo. La regla (no se debe decir «There's lots») no explica por qué el hablante puede hablar de esa manera; la generalización sí lo explica". Patricia Vining Lung y Janet A. De Cesaris, Investigación de gramática (Boston: Heinle \& Heinle, 1992) 2. 
acto comunicativo a la vez que refuercen determinadas funciones de lenguaje. Con este tratamiento de errores se evita pasar por alto las imprecisiones. Muchos estudiantes se lamentan de que sus profesores no les ayudan a corregir sus errores a tiempo y han tenido que luchar con la fosilización de usos incorrectos del idioma.

Luego de esta primera experiencia de investigación, informes y tratamiento de errores, los estudiantes ya estarán mejor preparados para las sucesivas, dos o tres a lo largo del curso. Debido a que los estudiantes del curso eligen temas diversos o repetidos aunque con perspectivas diferentes, suelen sentirse satisfechos al final del ciclo o semestre, pues además de mejorar el empleo del idioma y el conocimiento de la cultura, han tenido la oportunidad de comunicarse con personas diversas.

\section{La motivación de los estudiantes; condición «sine qua non»}

La condición sin la cual no es posible un apropiado desarrollo del curso ESL/LE con la cultura como eje transversal y con la metodología de ABT, es que los estudiantes estén motivados. No es posible contar con que todos muestren el mismo entusiasmo para empezar cualquier proyecto de investigación. Para ello, entre las pruebas de diagnóstico el profesor debe incluir instrumentos para conocer sus intereses y posibles rechazos. El mejor consejo para todo docente es no subestimar al alumno; incluso el estudiante que al principio luzca más reticente o apático podría dar muy agradables sorpresas en cuanto a la calidad de sus trabajos si se logra que se involucre en algo que realmente le interesa. Por ello, los temas de investigación no deben ser asignados de forma arbitraria.

La motivación puede propiciarse mediante conversaciones, películas, visitas a lugares, etc. No obstante, cualesquiera que sean estos recursos, deberán ser atractivos y apropiados para el nivel del estudiante y su sensibilidad. El docente debe conocer cada uno de ellos de antemano y evaluar sus ventajas y desventajas para solucionar cualquier inconveniente que se pudiera presentar. 


\section{¿Cómo guiar a los estudiantes?}

\section{En el trabajo de campo}

Es natural que los estudiantes que apenas ingresan al país desconozcan su realidad y requieran de mucha ayuda para identificar temas de interés en sus investigaciones. Para una mejor inserción de los alumnos en el trabajo de campo, tanto ellos como el docente deben tener plena conciencia de las dimensiones sociales del curso ESL/LE. Para reforzar la importancia de este punto, dice Brooks:

La construcción de normas sociales es un proceso de construir expectativas sobre cómo uno se debe comportar y lo que debe hacer...Los procesos de construcción y comunicación que se dan durante la clase de lengua extranjera, no sólo influyen en lo que ocurre en la clase, sino también en lo que se aprende. Es decir, a medida que los estudiantes aprenden las partes del idioma, aprenden también a ser miembros competentes de la clase y así participan en las actividades de aprendizaje... Al igual que ocurre al aprender la lengua materna, adquirir una lengua extranjera comporta un proceso tácito de socialización que se da mediante la interacción social ${ }^{8}$.

Ese proceso de socialización construido en clase, debe ser llevado a la comunidad misma, de la cual forma parte el centro educativo. A los alumnos se les ha de exhortar a que seleccionen temas sobre los cuales puedan indagar, entrevistándose con personas locales. El profesor puede hacer sugerencias en cuanto al vocabulario a emplear en las entrevistas, al modo de tratar con los entrevistados y con otras personas para que la información sea fiable. Al tiempo que los estudiantes aprenden contenidos acerca del tema investigado, socializan con la gente; esto les permite otros beneficios como la

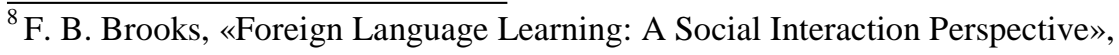
Patten y Lee, 17. 
ruptura de estereotipos mutuos y el ver de cerca las realidades del país en que están. En la elaboración del cuestionario, los estudiantes verifican con el profesor la propiedad de las preguntas planteadas. Además, conviene que el docente ayude en el caso de que los entrevistados empleen vocabulario desconocido para los estudiantes o ante cualquier situación particular que se presente. Por lo general, los estudiantes se muestran satisfechos de sus conversaciones con la gente local.

\section{En la elaboración de los informes}

Es posible que algunos alumnos sientan ansiedad y quieran que el docente les corrija los errores de sus informes, previo a la presentación en clase. Esto no parece conveniente, porque la idea es que los estudiantes muestren lo que pueden hacer por sí mismos y luego se entrará en la discusión de lo que hay que corregir.

Estas presentaciones se pueden programar una o dos veces más a lo largo del curso. Hay que tener en cuenta la distribución del tiempo para asignar el trabajo, realizarlo, presentar los informes y dar tratamiento a los errores. En las demás clases se puede trabajar en la

ejecución de tareas concretas, manteniendo la cohesión que da el eje transversal. El curso se programa en unidades de clase, distribuidas a los largo del número de semanas y conservando el distanciamiento proporcional entre las presentaciones orales y escritas; por ejemplo, el diagnóstico puede que ocupe la semana uno; la realización de la primera investigación, sus presentaciones y tratamiento de errores tomará dos o tres semanas más. La siguiente investigación se asignara a partir de la séptima semana. Es decir, se dispone de las semanas cinco y seis para desarrollar experiencias de aprendizaje a modo de unidades del curso que pueden requerir de una o dos lecciones cada una. 


\section{Ejemplo de una tarea asignada dentro de una unidad del curso}

El curso ESL/LE que se propone resguarda la dimensión cultural como eje transversal. La elección de temas de investigación, las entrevistas con personas locales, la presentación de informes orales y escritos y el tratamiento de errores sobre esos temas, forman lo medular del curso. Esas actividades se realizan dos o tres veces durante el curso. Entre tanto, se preparan lecciones que consistan en tareas cortas, realizables en una o dos sesiones en las que se practiquen contenidos concretos de lenguaje, a la vez que se mantenga la unidad con el eje transversal. A continuación se presenta la muestra de una unidad de aprendizaje dentro del curso.

Población meta: grupo intermedio, $\mathrm{B} 1^{9}$

Nacionalidades: Estados Unidos, Canadá, Alemania, Austria

Número de estudiantes: diez

Edades: entre 20 y 22

Contexto: Esta unidad se ubica dentro de un plan de cinco unidades y corresponde a la número 3. En la unidad anterior, se estudiaron preliminarmente los pronombres de objeto directo e indirecto. En la presente unidad se refrescan esas estructuras para establecer la base de la conjugación de verbos «de accidente», o sea los que se conjugan en razón del objeto.

\section{UNIDAD TRES: «USTED NO SABE LO QUE ME PASÓ UNA VEZ...»}

Meta de la tare $a^{10}$ : los estudiantes podrán narrar anécdotas que les hayan ocurrido por accidente, mediante el empleo de formas coloquiales que impliquen que la acción escapa al control del sujeto

${ }^{9} \mathrm{~B} 1:$ «Puede comprender los puntos principales de lo que se dice sobre temas concretos o abstractos expuestos en lenguaje claro y estándar, siempre que se trate de cosas familiares en el trabajo, la escuela, los momentos de ocio, etc. Puede desenvolverse en la mayor parte de las situaciones de un viaje por zonas en las que se habla la lengua que estudia. Puede producir un discurso sencillo y coherente sobre temas familiares de su interés. Puede contar sucesos, experiencias o sueños, expresar sus esperanzas y metas y justificar o aclarar brevemente opiniones o proyectos personales». MCEREL, «Escala global».

${ }^{10}$ Para ver la diferencia entre metas u objetivos, veamos lo que nos dice K. Graves [Designing Language Courses (Boston: Newbury House, 1996) 17]: «Goals are 
Tiempo: 2 horas, 30 minutos (no necesariamente consecutivas)

Lenguaje: pronombres reflexivos y de objeto directo e indirecto; verbos que, acompañados de pronombres indican situaciones accidentales: olvidar, quemar, perder, quedar, descomponer, etc.

Habilidades: producción oral, comprensión auditiva, escritura.

Estrategias: activación de conocimiento previo, negociación de significados, preguntas para clarificar, empleo de circunloquios.

I. M.: interpersonal, verbo-linguiística, visual-espacial

Materiales: pizarra, marcadores, láminas ilustrativas, hojas de trabajo con instrucciones escritas.

- ACTIVIDAd DE INICIO: La rutina de cada día... (20 minutos)

Objetivo: los estudiantes refrescarán el vocabulario de rutina diaria mediante el intercambio de ideas sobre lo que hacen en su rutina de cada día, utilizando correctamente algunos verbos reflexivos: levantarse, ducharse, vestirse, peinarse, etc.

Procedimiento:

1. Al ingresar en el aula, los estudiantes encontrarán en el centro del salón una mesa sobre la cual hay papeles cada uno con alguna de las siguientes palabras o expresiones que indican secuencia de eventos. Ej.: Primero, luego, después, a continuación, más tarde, posteriormente, al final, por último. El docente los invita a ponerlas en orden y fijarlas en el pizarrón.

2. El profesor les pide a los estudiantes formarse en parejas y les da estas instrucciones: «El estudiante A le contará a su compañero(a) cinco objetos que él o ella hace cada día antes de salir de casa; el estudiante B le contará a su compañero(a) cinco objetos que él o ella hace cada día al volver a casa». El profesor invita a los estudiantes a relatar ante la clase lo que escucharon de su compañero(a), teniendo en cuenta los términos de secuencia escritos en la pizarra.

3. El profesor controla la narración de los relatos y toma nota de las imprecisiones que escucha para proveer instrucción al final.

general statements of the overall long-term purposes of the course. Objectives Express the specific ways in which the goals will be achieved. The goals of a course represent the destination. The objectives the various points that chart the course toward the destination». 


\section{Antes de la tarea}

Comentario: El planteo de una tarea implica un equilibrio entre instrucción y práctica, a fin de que los estudiantes produzcan correctamente lenguaje. El ABT no parte de la instrucción sino de la práctica, ya que se espera que los alumnos adquieran la precisión a partir de la corrección de lo que ellos mismos han producido. Al asumir riesgos adquiriendo independencia. Por otra parte, ABT tampoco acepta la metodología de «nade o húndase», en la que se les impone a los estudiantes una tarea sin haberles dado las herramientas para realizarla. Por ello, luego de la actividad de inicio, que es un calentamiento, se desarrolla la tarea en tres etapas: antes, durante y después de la tarea. Para que esto funcione, el docente debe propiciar un ambiente adecuado. En el diseño de la tarea se prevén todas las condiciones y recursos necesarios para que todo se encuentre a disposición a la hora de ejecutar la tarea. En la etapa antes de la tarea, se realiza una serie de actividades que vayan dando preparación de lo más básico a lo más complejo. El número de actividades en esta etapa depende del número de funciones lingüísticas cuyo dominio el estudiante debe demostrar a fin de que se le considere apto para la realización de la tarea.

\section{- ACTIVIDAd 1: ¿Cómo elaboró su cuestionario para este proyecto? (5 minutos)}

Objetivo: Referirse oralmente al proceso de elaboración del cuestionario mediante el empleo correcto de pronombres de objeto directo.

Instrucciones:

1. Cuéntele a su compañero(a) cómo preparó su cuestionario para el proyecto. Se le sugieren verbos como: diseñar, escribir, completar, terminar, transmitir.

2. Cuéntele a la clase lo que le dijo su compañero(a).

- ACTIVIDAD 2: ¿Cómo resultaron sus entrevistas? (10 minutos)

Objetivo: Referirse oralmente a lo que ocurrió durante la etapa de las entrevistas, usando correctamente los pronombres de objeto indirecto y los verbos sugeridos.

Instrucciones: 
1. Cuéntele a su compañero(a) cómo fueron sus entrevistas para el proyecto. Se le sugieren verbos como preguntar, consultar, aclarar, dar, decir, contestar, repetir.

2. Cuéntele a la clase lo que le dijo su compañero(a).

\section{- ACTIVIDad 3: Efectuadas las entrevistas, ¿qué puede decirme sobre los ticos? (15 minutos)}

Objetivo: Expresar oralmente una opinión acerca de los entrevistados, utilizando correctamente verbos que siguen la conjugación de «gustar». Instrucciones:

1. Cuéntele a su compañero(a) algo de lo que aprendió de los ticos por medio de sus entrevistas. Se le sugiere emplear verbos como agradar, gustar, encantar, incomodar, molestar, parecer, caer bien, enojar.

2. Cuéntele a la clase lo que le dijo su compañero(a).

\section{- ACTIVIDAd 4: Esta es mi excusa: «Se me olvidó la fecha de tu cumpleaños» (30 minutos)}

Objetivo: Los estudiantes reconocerán la estructura de un formante verbal que indica que determinada acción se dio accidentalmente; esto lo harán mediante el contraste de esa estructura con otra que indica que determinada acción se hizo con intención.

Procedimiento:

1. En parejas, los estudiantes reciben un juego de láminas entremezcladas; en cada una de ellas figura una ilustración y una leyenda como las siguientes: «Quemé las cartas de mi exnovia», «A mi mamá se le quemó el pan», «Yo olvidé a mi exnovio(a)», «A mí se me olvidó la fecha de su cumpleaños», etc.

2. El profesor pide a los estudiantes que agrupen las láminas en pares, encontrando cada par que lleva el mismo verbo.

3. Los estudiantes deben analizar el formante verbal de cada lámina de cada par: qué significa el verbo, qué pronombres lleva, cómo están colocados los pronombres en relación al verbo, etc.

4. El profesor pide a algunos estudiantes que, por turnos, pasen a la pizarra a explicar: a. En cada par, cuál acción es accidental y cuál es intencional; b. en cuáles acciones el verbo está conjugado de acuerdo con el sujeto (yo quemé las cartas...) y en cuáles acciones el verbo está conjugado de acuerdo con el 
objeto (a mi mamá se le quemó el pan); y c. Cómo es la conjugación de un verbo en razón del objeto, en cada una de las personas gramaticales.

5. El profesor entrega a cada estudiante una lista de verbos factibles de conjugar en razón del objeto: quemar, quebrar, olvidar, perder, etc. Se practican diferentes ejemplos y se aclara lo que cada verbo indica exactamente. Esta actividad puede extenderse un poco en el tiempo, pues es la médula de la tarea que sigue.

N.B. Las cuatro actividades anteriores están muy dirigidas y controladas. El profesor está encaminando el proceso a un objetivo muy concreto: La producción de determinado patrón de lenguaje en la comunicación. Se prevé cada detalle de lo que se necesita para alcanzarlo. Esta es exactamente la función de esta etapa. Si los estudiantes yerran en la precisión de lo que producen, habría que detectar el error en lo que pudo andar mal aquí.

\section{Durante la tarea: Usted no sabe lo que una vez me ocurrió... (30 minutos)}

Comentario: Esta etapa durante de la tarea es la medular de todo el trabajo. Todo lo hecho ha estado encaminado a que los alumnos den este paso. Si la preparación ha sido la adecuada, se verá reflejada ahora. Lo mismo que los errores deberán corregirse analizando su origen en las actividades anteriores.

Objetivo: Los estudiantes presentarán ante la clase un resumen de lo que oyeron de su compañero(a), mediante el empleo correcto de verbos conjugados en razón del objeto.

Procedimiento:

1. En parejas, los estudiantes reciben una hoja con la siguiente instrucción: «Cuéntele a su compañero(a) acerca de algunas situaciones desastrosas, patéticas o chistosas que le han ocurrido a usted en su vida. Use verbos como romper, ensuciar, arrugar, derramar, descomponer, quemar, quedar, romper, quebrar, etc. Recuerde que sus verbos deben dar la connotación de acciones accidentales y no intencionales (según el hablante nativo, no fue algo que usted hizo, sino algo que a usted le pasó)».

2. Mientras los estudiantes escuchan a su compañero(a), toman nota para exponer después. El profesor pedirá esta vez a todos que expongan lo que oyeron de sus compañero(a). 
3. El profesor dará instrucción sobre los errores detectados durante la exposición.

\section{Después de la tarea:}

\section{- Actividad 1: Aquí esta su informe escrito del proyecto. No lo} entregue aún al profesor. Démoselo juntos más tarde.

Objetivo: Los estudiantes reforzarán su conocimiento sobre de la posición de los pronombres de objeto con relación al verbo, mediante el análisis de algunos ejemplos.

Procedimiento. El profesor escribe en la pizarra las siguientes frases: «Ya terminé el proyecto. Se lo daré al profesor», «Debo dárselo al profesor hoy mismo», «Se lo debo dar al profesor cuanto antes», «Déselo al profesor», «No se lo dé al profesor todavía», «Antes/después de dárselo al profesor, yo...». El profesor pide a los estudiantes que observen la particularidad de cada ejemplo para extraer de cada cual una regla para ubicar la posición de los pronombres de objeto con relación al verbo.

\section{- ACTIVIDAd 2: Se me quedó la tarea en la casa (TAREA)}

Objetivo: Los estudiantes reconocerán el significado que pueden tener algunos verbos mediante la confección de oraciones con ayuda de hablantes nativos.

Procedimiento:

1. El profesor entrega a cada estudiante una hoja con los siguientes formantes verbales:

\begin{tabular}{|l|l|}
\hline Queda: & Yo quemo: \\
A mí me queda: & Ella se quemó: \\
Yo me quedo: & A mí me quemó ella: \\
Se me quedó: & A ella se le quemó: \\
\hline
\end{tabular}

2. La hoja contendrá además esta instrucción: «Pídale a algún hablante nativo que le ayude a completar oraciones con estos formantes verbales». 3. En la siguiente lección, se revisarán las oraciones en clase y se comentará sobre los diferentes significados y connotaciones que pueden tener. 


\section{Conclusión}

Al sistematizar la experiencia de la enseñanza del español como segunda lengua en varios de los programas adscritos al Departamento de Estudios Hispánicos de la Universidad Nacional, podemos señalar algunos alcances. Se resalta no sólo la oportunidad sino también la urgencia de ofrecer este aporte a los nuevos profesores pues, como se ha dicho, los docentes actuales hemos sido pioneros en organizar los programas, diseñar los cursos, desarrollar e implementar los materiales, evaluar las fortalezas y debilidades de lo que se hace y lo más importante, centrar la experiencia de aprendizaje en torno a ejes temáticos, de los cuales, en nuestro caso se ha optado por el tema de la cultura.

La selección del tema obedece a la necesidad de acercar a los estudiantes internacionales y a la población local hacia una relación capaz de contribuir en la superación de prejuicios y estereotipos. Esta ha sido la visión y la misión de los cursos de español que han inspirado la preparación de este artículo. Nuestros estudiantes adquieren conciencia de la diferencia entre un estudiante internacional y un turista. Este último suele interesarse por el placer que el país puede ofrecerle. Aquél se interesa por el país en sí y todo lo que en él converge. Investiga sobre asuntos culturales y sociales y les muestra a los habitantes de la comunidad que su permanencia entre ellos tiene una intención mucho más benéfica para ambas partes. El ciudadano local comprende que los foráneos no entran necesariamente en el esquema preconcebido que nos han labrado los medios; el estudiante aprende a respetar la cultura local y a percibir el otro como a un igual, como a un amigo, como a un sujeto de derechos y aspiraciones igual que él. 